\title{
REVIEW
}

\section{Glutamine supplementation to critically ill patients?}

\begin{abstract}
Jan Wernerman
This article is one of ten reviews selected from the Annual Update in Intensive Care and Emergency Medicine 2014 and co-published as a series in Critical Care. Other articles in the series can be found online at http://ccforum.com/series/annualupdate2014. Further information about the Annual Update in Intensive Care and Emergency Medicine is available from http://www.springer.com/series/8901.
\end{abstract}

\section{Introduction}

A recent study (Reducing Deaths due to Oxidative Stress, REDOXS) reported harm in critically ill patients who received glutamine supplementation [1]. This is in contrast to a number of earlier studies reporting beneficial effects or failing to demonstrate any effect [2], [3]. Naturally, this finding raises a number of questions, which are not answered by combining all existing studies into a large meta-analysis. This overview will discuss existing clinical data, including dosing and selection of patients. In addition, suggested mechanisms will be discussed from a clinical perspective. Finally, possibilities for future research will be outlined.

\section{Rationale for supplementation}

The background to the suggestion that critically ill patients should receive glutamine supplementation is that plasma glutamine concentration at intensive care unit (ICU) admission is an independent predictor of an unfavorable outcome [4], [5]. Empirically, a plasma concentration of $420 \mu \mathrm{mol} / \mathrm{l}$ has repeatedly been reported as a cut-off for a low plasma glutamine concentration associated with a higher risk of mortality in adults [4], [5]. In principle, the same effect applies in critically ill pediatric patients, but here the low mortality rates have not made it possible to demonstrate a mortality disadvantage, although a morbidity disadvantage has been reported [6]. Approximately one third of ICU admissions are consistently found to have a low plasma glutamine concentration, and this is independent from conventional risk-scoring [1], [4]-[6]. In a study from Stockholm, the mortality associated with a low ICU admission glutamine concentration was to a large extent due to the post-ICU mortality within 6 months from ICU admission [5].

In addition to the predictive value of a low plasma glutamine concentration at ICU admission for an

*Correspondence: jan.wernerman@karolinska.se

Department of Anesthesia and Intensive Care Medicine, Karolinska University

Hospital, Huddinge, Stockholm, Sweden unfavorable outcome, there seem to be a similar prediction also for high plasma glutamine concentrations at admission [5]. This group of patients, however, is much smaller, and the evidence for this prediction is mostly in form of case series. It has been reported that acute liver failure is quite often associated with high or very high plasma glutamine concentrations [7]. Chronic liver insufficiency and acute-on-chronic liver failure are not accompanied by high plasma glutamine concentrations. In single cases, it has been observed that terminal patients with multiple organ failure (not necessarily including advanced liver failure) have very high plasma glutamine concentrations. One can speculate that this observation may relate to impaired cellular integrity in general.

In parallel to the association between a low plasma glutamine and an unfavorable outcome, there is an extensive literature about the essential role of glutamine in a number of experimental systems, including whole animals. Cell division demands an increase in nucleotide production, and glutamine is a main precursor for this type of synthesis. Cell culture media usually contain a much higher free glutamine concentration than does human plasma, and lowering of glutamine concentration in cell culture media is associated with a lower rate of cell division. Many cultured cells prefer glutamine over glucose as their main energy source, and imposing stressful events to the cell culture is reported to enhance the preference for glutamine over glucose as energy substrate. In tissues and whole animals, it is the rapidly replicating cells that seem to be particularly dependent on glutamine availability. Enterocytes in the gastrointestinal tract and immune-competent cells are reported to be particularly sensitive to glutamine depletion. Histological changes and bacterial translocation in the gut occur when there is glutamine shortage, and provision of glutamine can reverse this effect. Similarly, for immune-competent cells, markers of immune function deteriorate during glutamine shortage, to return back to normal upon restoration of glutamine availability. 


\section{Targets for supplementation}

With this background, the suggestion that glutamine shortage should be compensated for by supplementation is not far-fetched and consequently a number of clinical studies have been performed, mainly in critically ill patients. Behind the suggestion to supplement there are two different philosophies: To substitute a deficiency or to administer a pharmacological agent (pharmaconutrition). It is recommended that these two philosophies be separated because the target for treatment is different in the two cases. To supplement to normal levels would mean adding supplementation in order to reach normal plasma concentrations, or normal availability in tissues if more invasive monitoring is possible. To administer a pharmaconutrition agent on the other hand, would mean that a dose-response relation is presumed and that the desired response effect can be defined. This effect may be in terms of plasma glutamine concentrations, but may also be in terms of other measureable effects related to glutamine intake.

When determining the target for dosing of exogenous glutamine supplementation, small studies of intravenous supplementation restoring hypoglutaminemia back to normoglutaminemia are usually cited [8], [9]. These data have also been applied to enteral administration of supplementation, although enteral doses only marginally affect plasma concentration [10]-[12]. If the pharmaconutrition philosophy is applied, higher doses are usually given, although the rationale for doses beyond what may be needed to restore a low plasma concentration is purely hypothetical. Finally, the effect of treatment on glutamine availability is only very rarely defined in the available clinical studies. At best, articles report the success of administering the intended dose, but sometimes not even that is communicated in the article.

Available data make it highly unlikely that all critically ill patients are depleted in glutamine, as only approximately one third of patients admitted to the ICU have a plasma glutamine concentration $<420 \mu \mathrm{mol} / \mathrm{l}$. Furthermore, a higher risk of an unfavorable outcome indicated by a high APACHE II score or a high sequential organ failure assessment (SOFA) score is not statistically associated with glutamine depletion. In contrast, glutamine depletion at admission is not associated with risk scoring [4], [5]. However, the risk for a given patient may be increased if a low admission glutamine concentration is present (Figure 1). As pointed out by Rodas et al. this additional risk may be most pronounced for the group of patients with a moderate mortality risk [5]. If patient recruitment in a study focuses on patients with high mortality risk or low mortality risk, the additional risk associated with concomitant glutamine depletion will be less pronounced.

If exogenous glutamine supplementation aims to compensate for an additional risk imposed on patients

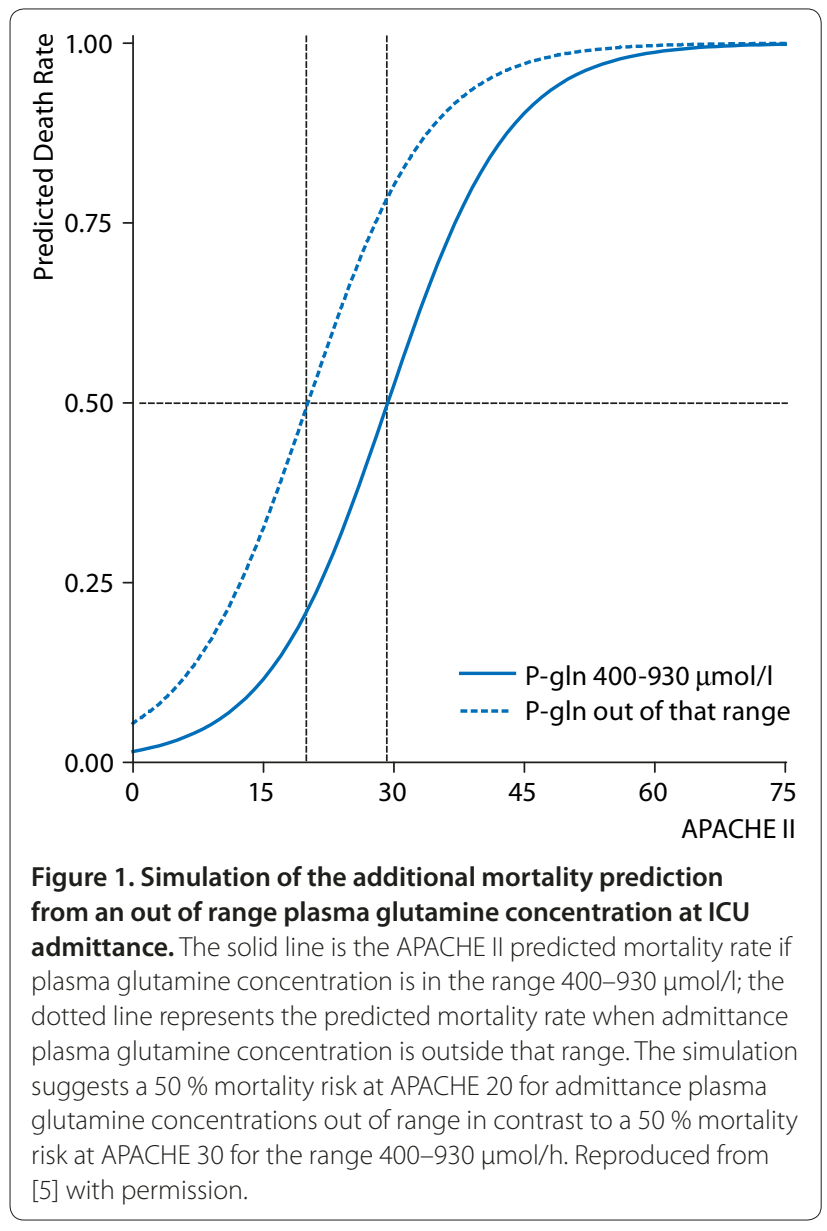

with glutamine depletion, the target group should be critically ill patients with a moderate mortality risk, with low admission plasma glutamine concentration, who are in need of nutrition support for, for example, not less than 5 days. The more a study population deviates from this target group in which a beneficial effect can be hypothesized, the less likely it will be that the study will show an effect of exogenous glutamine supplementation attributable to glutamine supplementation.

If, on the other hand, the hypothesis is that a pharmacological provision of glutamine may be beneficial, the target group should be patients with a high mortality risk with a pathology likely to respond to effects associated with providing a surplus of glutamine. To just combine all available studies of glutamine supplementation in critically ill patients in a meta-analysis will not contribute to answering the question whether exogenous glutamine supplementation may be beneficial or may cause harm. It should be recognized that different studies are based on very different assumptions of the involved mechanisms.

Additional information on glutamine status in the individual patient, in addition to the plasma concentration, can be obtained from studies about glutamine turnover. Available techniques use isotopically-labeled 
glutamine to estimate the endogenous rate of appearance of glutamine. A limited number of studies have been published employing this type of technique. Available data show that the endogenous production of glutamine is in the region of $50-80 \mathrm{~g} /$ day in adults [13]-[16]. Furthermore, in healthy individuals the endogenous rate of appearance is higher in the fed state as compared to the postabsorptive state [13], [14], reflecting that intake of amino acids other than glutamine stimulates glutamine synthesis de novo mainly in skeletal muscle. The possible relationship between plasma glutamine concentration, endogenous rate of appearance for glutamine, and outcome in critical illness has so far not been defined. In healthy individuals, as well as in critically ill patients, it has been demonstrated that exogenous glutamine supplementation does not decrease the endogenous rate of appearance, which suggest that there is not a negative feed-back mechanism to decrease de novo glutamine synthesis when concentration is high [15], [17]. The absence of such a negative feed-back may be related to the fact that glutamine is an inter-organ transporter of nitrogen from the periphery to the liver. The alternative, free ammonia, is potentially neurotoxic, which glutamine is not. Therefore, it is most likely metabolically sound to synthesize glutamine for this transportation and, in the liver, a surplus of nitrogen will be converted into urea to be eliminated in the urine. These pathways may be compromised in critically ill patients with failure of these organs. Studies of glutamine turnover and elimination in critically ill patients with compromised liver and/or kidney function are, however, not available. To better define the possible role of exogenous glutamine supplementation, more information from studies of glutamine kinetics and turnover are, therefore, needed.

\section{Which patients are suitable for glutamine supplementation?}

The initial studies of glutamine supplementation were small and involved patients who were receiving parenteral nutrition [18], [19]. Results were encouraging in terms of outcome benefits, and although the generalizability of these studies was limited, meta-analyses including these studies indicated mortality benefits and guidelines recommended intravenous supplementation of glutamine when parenteral nutrition was given to critically ill patients [20], [21]. When glutamine was given enterally, the results were much less conclusive, and meta-analyses of these results were not able to demonstrate beneficial effects; consequently guidelines did not recommend enteral glutamine supplementation [20], [22]. Part of this documentation refers to trials that used so-called immune-nutrition, meaning that a number of agents with potential effect on immune function were combined in the supplementation. It goes without saying that the results from such trials do not allow discrimination of the possible contributions for the individual ingredients in the mixtures given. Nevertheless, when given to critically ill patients receiving enteral nutrition, generally beneficial effects have not been demonstrated when glutamine has been the sole supplement or part of a supplemented mixture [2], [3].

It is unfortunate when reviewing the literature that selection of patients, target for dosing, and effect of treatment are often not very well motivated. Critically ill patients included in studies of exogenous glutamine supplementation are usually patients admitted to the ICU, sometimes confined to only those receiving mechanical ventilation. Often a dichotomization is made related to the route of nutrition administration. There is some empiric evidence for such a discrimination [23], [24], but the possible underlying mechanisms are obscure. Risk-scoring of patients at admission or sequentially during the ICU stay does not usually include any estimate of gut function. It is again empiric evidence that successful enteral feeding, indicative of a functional intestinal tract, is associated with a more favorable outcome, also in patients with comparable risk-scoring (only reflecting other organ systems) [25]. Hence, when evaluating the literature it is important to consider that the scales in the risk-scoring instruments are not linear and that the scores for selected subgroups of patients may not directly correspond to the prediction for unselected patient populations for whom the scoring systems were validated.

In this context, there is also reason to comment upon the length of treatment period. In general there is a marked difference between the mean and the median lengths of stay in the ICU. This difference reflects that the vast majority of patients are short-stayers, less than 45 days, while a minority of patients stay for a long time in the ICU. Nevertheless, this small group of long-stayers consumes the majority of ICU days in a given unit. The variability in length of ICU stay within the patient population constitutes a problem when inclusion and exclusion criteria are to be defined. Is it feasible that exogenous supplementation to a critically ill patient for 2 days will make a difference?; 3 days?; 5 days?; 7 days? On the other hand, it is often very difficult to foresee the length of stay for the individual patient at ICU admission. It may, therefore, be reasonable to have a predefined subgroup analysis of those patients who are given treatment for a period of time that is found sufficient for an effect to be demonstrated.

\section{Comments on recent studies with glutamine supplementation}

With this background, it is quite clear that studies including patients with a high mortality risk, who are 
poorly fed and supplied with pharmacological doses of glutamine are not very likely to produce results on how glutamine supplementation may be beneficial for critically ill patients. Indeed, this strategy turned out to be harmful, possibly attributable to the pharmacological doses of glutamine used [1]. Because glutamine supplementation given to the right patients in the right doses may be helpful, this result imposes the risk of 'killing' the glutamine supplementation concept. To just look upon the critically ill patient as a 'black box', and to pour in pharmacological doses of glutamine without proper nutritional support and without knowledge of whether or not glutamine deficiency is present is obviously not a very good idea [26].

A neutral or non-conclusive effect, which was the result of the Scottish glutamine study, would have been the logical result also in the REDOXS study; therefore, a number of important differences between these studies should be highlighted. The Scottish SIGNET study included well-fed patients with a pragmatic protocol [27]. The study has been criticized for the relatively low dose of glutamine given, for not communicating how much of the intended dose the patients were actually given, and for the limited time period of treatment. The REDOXS study, on the other hand, was not neutral in terms of outcome, rather it demonstrated harm [1]. It is the first study to demonstrate harm related to provision of exogenous glutamine in critically ill patients. Earlier a report of possible harm was published in a case series of hematological patients with insufficient comparability between patients who received extra glutamine and those who did not [28].

A closer look at the comparability of patients in the REDOXS study, also reveals a difference between the patient groups that may offer an alternative explanation for the result of harm. The inclusion criterion was patients with $\geq 2$ organ failures according to SOFA scoring, and the group given glutamine had a similar mean SOFA to the group not given glutamine; so far so good. However, if the two groups are compared in patients with $>2$ organ failures, suddenly there is a statistically significant difference between them (Figure 2). To disregard this finding, the risk prediction by SOFA scoring must be assumed to be linear, but there is actually no evidence for such a linearity. On the contrary, there is evidence that mortality rate increases logarithmically with the number of organ failures [29]. Actually the level of statistical significance attributable to the skewed distribution of patients with $>2$ organ failures is larger than the level of significance for the harm of pharmacological doses of glutamine. This is not a scientific comparison, but the investigators of the REDOXS study could perform a multiple stepwise regression analysis to address this possible bias in patient selection.

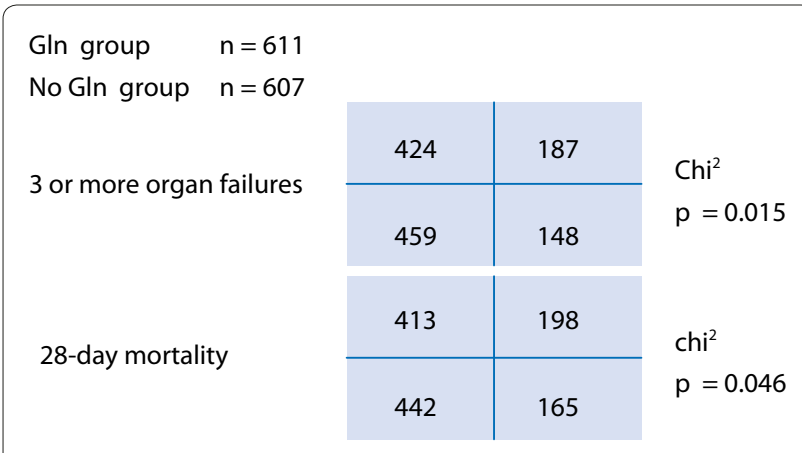

Figure 2. Calculations of the likelihood of occurrence of three or more organ failures and of 30-day mortality rate in the REDOXS study related to whether randomized to glutamine (GLN) supplementation or not [1].

If the increased mortality in the glutamine group of the REDOXS study can be associated with a difference between the two groups other than the glutamine supply, we are suddenly in quite a different position. The expected non-conclusive result of the study, according to the chosen study population, would then no longer disqualify studies with protocols aimed at evaluating supplementation of an actual glutamine deficiency. If on the other hand, no other difference than the supply of 50 g glutamine daily can be found between the two groups in REDOXS, the mechanism behind the increased mortality must be looked for. Could short-term use of a bodily substance lead to intoxication? At this time-point, we do not know whether the patients that died in the REDOXS study did so with high glutamine concentrations. In the small subgroup of patients in REDOXS in which plasma glutamine concentrations were recorded, no information over outcome was communicated. The endogenous production of glutamine is some $50-80 \mathrm{~g} / 24 \mathrm{~h}$; will an exogenous provision of similar magnitude cause toxicity? This effect has been reported in patients with liver encephalopathy, but in that situation the effect is not specific for glutamine but is reported for amino acids or proteins in general when given at high doses [30].

\section{Suggestions for future research}

It is obvious that more studies are needed, but it may not currently be possible to randomize patients to be given extra glutamine. At least, it cannot be considered ethical to give glutamine supplementation without monitoring plasma concentrations. However, quite large doses can be given enterally with only marginal effect on plasma concentration. Still toxic? Obviously more observational studies are needed and in addition the toxicity of glutamine must be characterized. Is the toxicity related to plasma concentration?

In general, provision of glutamine by the enteral route only marginally influences plasma concentration, 
whereas intravenous provision immediately leads to an increased plasma concentration [8]. Moreover, with a high rate of intravenous glutamine infusion, a steady state will be reached [8], [9]. The suggested relationship between a high plasma glutamine concentration at admission and an unfavorable outcome is based on a very limited number of observations, and needs to be further explored by observational studies. The connection between a high plasma glutamine level and acute fulminant hepatic insufficiency is well known [7], but whether a relation between glutamine level and outcome in this specific group exists is not known [30]. In chronic liver disease and in acute-on-chronic liver failure, high plasma glutamine concentrations are not observed. The manufacturer of glutamine containing dipeptides for intravenous use does not recommend supplementation to patients with liver and/or kidney insufficiency. This recommendation is mainly based on the very limited documentation for this patient group, rather than actual data about glutamine toxicity in liver failure or kidney failure. The relationship between kidney failure and mortality in REDOXS is more difficult to interpret [1]. As kidney failure is most often the third organ failure, increased mortality is seen with kidney failure in critical illness. Again we come back to the possibility of a skewed distribution between the randomized groups in that particular study.

Can clinical use of glutamine supplementation be recommended today after REDOXS? Clearly not to critically ill patients with two (or three) organ failures in the acute phase, but what about other situations? It is speculated that the route of glutamine administration, enteral or parenteral, should make a difference. The handling of glutamine is clearly different with different routes of administration as reflected by the effect on plasma concentration [8], [12], but is possible toxicity related to route of administration? It can also be speculated that it is the pharmacological dose of glutamine that is harmful. Are moderate doses safe? These doses were used in the Scottish SIGNET study as well as in the Scandinavian glutamine study [27], [31]. Is glutamine combined with parenteral nutrition safe, while combination with enteral nutrition is harmful? This has recently been argued in meta-analyses in which published studies are divided according to how patients were fed [2], but is there a mechanistic rational for such a suggestion?

\section{Conclusions}

The result of the REDOXS study raises concern about whether or not large doses of glutamine can cause harm in critically ill patients. A number of peculiarities in the protocol of the study and in the patient selection should be clarified. This clarification can be done by the
REDOXS investigators and by observational studies of critically ill patients. In addition, more research is needed to characterize the relation between endogenous production of glutamine and exogenous supplementation of glutamine in critically ill patients. Finally, glutamine substitution to normalize plasma glutamine concentrations in critically ill patients should be evaluated in well-conducted randomized controlled trials. Meanwhile, it is recommended that use of glutamine supplementation be restricted to situations in which a safety protocol is used or within clinical studies.

\section{List of abbreviations used}

ICU: intensive care unit; GLN: glutamine; REDOXS: reducing deaths due to oxidative stress; SOFA: sequential organ failure assessment.

\section{Competing interests}

The author received reimbursements from participating in advisory boards from Baxter, Danone, Fresenius, and Nestlé. No financiation for this article. Academic, Pl of the Scandinavian Glutamine Study.

\section{Declarations}

Publication of this article was funded by grants within Karolinska Institutet.

\section{Published: 18 March 2014}

\section{References}

1. Heyland D, Muscedere J, Wischmeyer PE, Cook D, Jones G, Albert M, Elke G, Berger MM, Day AG, Canadian Critical Care Trials Group: A randomized trial of glutamine and antioxidants in critically ill patients. N Engl J Med 2013, 368:1489-1497.

2. Bollhalder L, Pfeil AM, Tomonaga Y, Schwenkglenks M: A systematic literature review and meta-analysis of randomized clinical trials of parenteral glutamine supplementation. Clin Nutr 2013, 32:213-223.

3. Novak F, Heyland DK, Avenell A, Drover JW, Su X: Glutamine supplementation in serious illness: a systematic review of the evidence. Crit Care Med 2002, 30:2022-2029

4. Oudemans-van Straaten HM, Bosman RJ, Treskes M, van der Spoel HJ, Zandstra DF: Plasma glutamine depletion and patient outcome in acute ICU admissions. Intensive Care Med 2001, 27:84-90.

5. Rodas PC, Rooyackers O, Hebert C, Norberg A, Wernerman J: Glutamine and glutathione at ICU admission in relation to outcome. Clin Sci (Lond) 2012, 122:591-597.

6. Ekmark L, Rooyackers O, Werneman J, Flaring U: Plasma glutamine correlates with multiple organ failure in critically ill children. Clin Nutr Supp/ 2009, 4:174 (abstract).

7. Clemmesen JO, Kondrup J, Ott P: Splanchnic and leg exchange of amino acids and ammonia in acute liver failure. Gastroenterology 2000, 118:1131-1139.

8. Berg A, Rooyackers O, Norberg A, Wernerman J: Elimination kinetics of L-alanyl-L-glutamine in ICU patients. Amino Acids 2005, 29:221-228.

9. Tjader I, Rooyackers O, Forsberg AM, Vesali RF, Garlick PJ, Wernerman J: Effects on skeletal muscle of intravenous glutamine supplementation to ICU patients. Intensive Care Med 2004, 30:266-275.

10. Houdijk AP, Rijnsburger ER, Jansen J, Wesdorp RI, Weiss JK, McCamish MA, Teerlink T, Meuwissen SG, Haarman HJ, Thijs LG, van Leeuwen PA: Randomised trial of glutamine-enriched enteral nutrition on infectious morbidity in patients with multiple trauma. Lancet 1998, 352:772-776.

11. Dechelotte P, Darmaun D, Rongier M, Hecketsweiler B, Rigal O, Desjeux JF: Absorption and metabolic effects of enterally administered glutamine in humans. Am J Physio/ 1991, 260:G677-G682.

12. Melis GC, Boelens PG, van der Sijp JR, Popovici T, De Bandt JP, Cynober L, van Leeuwen PA: The feeding route (enteral or parenteral) affects the plasma response of the dipeptide Ala-Gln and the amino acids glutamine, citrulline and arginine, with the administration of Ala-Gln in preoperative patients. Br J Nutr 2005, 94:19-26.

13. Darmaun D, Matthews DE, Bier DM: Glutamine and glutamate kinetics in humans. Am J Physiol 1986, 251:E117-E126. 
14. Matthews DE, Marano MA, Campbell RG: Splanchnic bed utilization of glutamine and glutamic acid in humans. Am J Physiol 1993, 264:E848-E854.

15. Rooyackers O, Prohn M, Van Riel N, Wernerman J: Bolus injection on 13 C-glutamine to study glutamine metabolism in humans. Clin Nutr 2005, 24:575-576.

16. van Acker BA, Hulsewe KW, Wagenmakers AJ, Soeters PB, von Meyenfeldt MF: Glutamine appearance rate in plasma is not increased after gastrointestinal surgery in humans. J Nutr 2000, 130:1566-1571.

17. van Acker BA, Hulsewe KW, Wagenmakers AJ, von Meyenfeldt MF, Soeters PB: Response of glutamine metabolism to glutamine-supplemented parenteral nutrition. Am J Clin Nutr 2000, 72:790-795.

18. Goeters C, Wenn A, Mertes N, Wempe C, Van Aken H, Stehle P, Bone HG: Parenteral L-alanyl-L-glutamine improves 6-month outcome in critically ill patients. Crit Care Med 2002, 30:2032-2037.

19. Griffiths RD, Jones C, Palmer TE: Six-month outcome of critically ill patients given glutamine-supplemented parenteral nutrition. Nutrition 1997, 13:295-302.

20. McClave SA, Martindale RG, Vanek VW, McCarthy M, Roberts P, Taylor B, Ochoa JB, Napolitano L, Cresci G, ASPEN Board of Directors, American College of Critical Care Medicine, Society of Critical Care Medicine: Guidelines for the provision and assessment of nutrition support therapy in the adult critically ill patient: Society of Critical Care Medicine (SCCM) and American Society for Parenteral and Enteral Nutrition (A.S.P.E.N.). JPEN J Parenter Enteral Nutr 2009, 33:277-316.

21. Singer $P$, Berger MM, Van den Berghe G, Biolo G, Calder P, Forbes A, Griffiths $R$, Kreyman G, Leverve X, Pichard C, ESPEN: ESPEN Guidelines on Parenteral Nutrition: intensive care. Clin Nutr 2009, 28:387-400.

22. Kreymann KG, Berger MM, Deutz NE, Hiesmayr M, Jolliet P, Kazandjiev G, Nitenberg G, van den Berghe G, Wernerman J, DGEM (German Society for Nutritional Medicine), Ebner C, Hartl W, Heymann C, Spies C, ESPEN (European Society for Paternal and Enteral Nutrition): ESPEN Guidelines on Enteral Nutrition: Intensive care. Clin Nutr 2006, 25:210-223.

23. Doig GS, Heighes PT, Simpson F, Sweetman EA, Davies AR: Early enteral nutrition, provided within $24 \mathrm{~h}$ of injury or intensive care unit admission, significantly reduces mortality in critically ill patients: a meta-analysis of randomised controlled trials. Intensive Care Med 2009, 35:2018-2027.
24. Heyland DK, MacDonald S, Keefe L, Drover JW: Total parenteral nutrition in the critically ill patient: a meta-analysis. JAMA 1998, 280:2013-2019.

25. Doig GS, Heighes PT, Simpson F, Sweetman EA: Early enteral nutrition reduces mortality in trauma patients requiring intensive care: a metaanalysis of randomised controlled trials. Injury 2011, 42:50-56.

26. Preiser JC, Wernerman J: REDOXS: Important answers, many more questions raised! JPEN J Parenter Enteral Nutr 2013, 37:566-567.

27. Andrews PJ, Avenell A, Noble DW, Campbell MK, Croal BL, Simpson WG, Vale LD, Battison CG, Jenkinson DJ, Cook JA, Scottish Intensive care Glutamine or seleNium Evaluative Trial Trials Group: Randomised trial of glutamine, selenium, or both, to supplement parenteral nutrition for critically ill patients. BMJ 2011, 342:d1542.

28. Pytlik R, Benes P, Patorkova M, Chocenská E, Gregora E, Procházka B, Kozák T: Standardized parenteral alanyl-glutamine dipeptide supplementation is not beneficial in autologous transplant patients: a randomized, doubleblind, placebo controlled study. Bone Marrow Transplant 2002, 30:953-961.

29. Moreno R, Vincent JL, Matos R, Mendonça A, Cantraine F, Thijs L, Takala J, Sprung $C$, Antonelli $M$, Bruining $H$, Willatts $S$ : The use of maximum SOFA score to quantify organ dysfunction/failure in intensive care. Results of a prospective, multicentre study. Working Group on Sepsis related Problems of the ESICM. Intensive Care Med 1999, 25:686-696.

30. Amodio P, Bemeur C, Butterworth R, Cordoba J, Kato A, Montagnese S, Ribe $M$, Vilstrup H, Morgan MY: The nutritional management of hepatic encephalopathy in patients with cirrhosis: International Society for Hepatic Encephalopathy and Nitrogen Metabolism Consensus. Hepatology 2013, 58:325-336.

31. Wernerman J, Kirketeig T, Andersson B, Berthelson H, Ersson A, Friberg H, Guttormsen AB, Hendriks S, Pettilä V, Rossi P, Sjöberg F, Winsö O, Scandinavian Critical Care Trials Group: Scandinavian glutamine trial: a pragmatic multicentre randomised clinical trial of intensive care unit patients. Acta Anaesthesio/ Scand 2011, 55:812-818.

doi:10.1186/cc13781

Cite this article as: Wernerman J: Glutamine supplementation to critically ill patients? Critical Care 2014, 18:214 